\title{
ApoC3 fires up monocytes to promote tissue damage
}

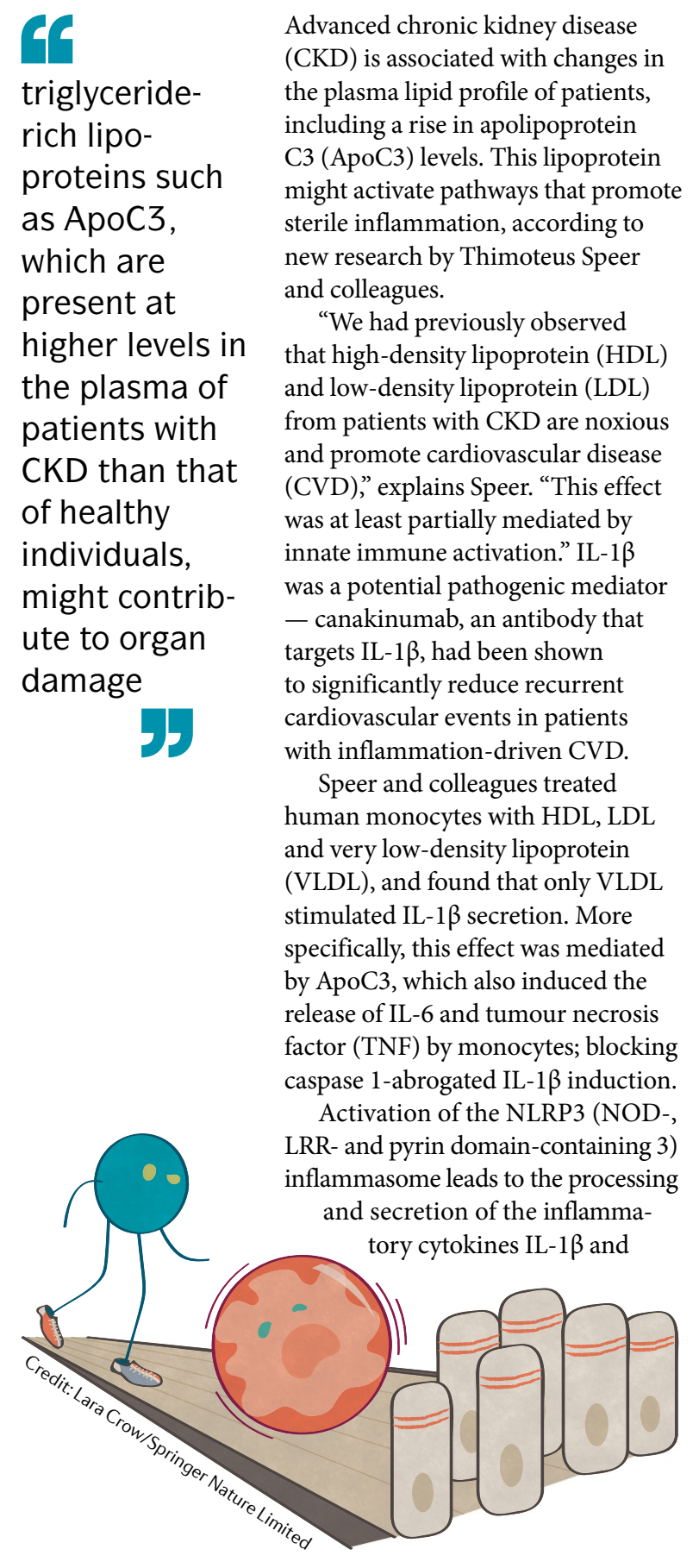

IL-18, in a process that involves caspase 1 activation. The researchers thus suspected that NLRP3, which functions as an intracellular sensor of exogenous and endogenous ligands associated with cellular stress, might be involved in the monocyte response to ApoC3. Although classical NLRP3 activation induces pyroptosis and is dependent on cellular potassium influx, neither of these effects were observed in monocytes exposed to ApoC3. Instead, this lipoprotein seemed to induce alternative NLRP3 activation, which was dependent on signalling through Toll/interleukin-1 receptor domain-containing adapterinducing interferon- $\beta$ (TRIF) and receptor-interacting serine/threonineprotein kinase 1 (RIPK1), as well as caspase 8 activity.

ApoC3 induced IL- $1 \beta$ secretion in human monocytes independently of ApoC3 internalization, suggesting the presence of a surface receptor. In human embryonic kidney (HEK) cells transfected with TLR2 and TLR4, ApoC3 induced NF- $\kappa B$ activation via TLR4, which seemed to heterodimerize with TLR2. Culture of human monocytes with ApoC3 also triggered calcium influx and increased superoxide production. Accordingly, IL-1 $\beta$ release was inhibited in the presence of calcium chelators or following inhibition of transient receptor potential cation channel subfamily M member 2 (TRPM2), known to have a role in the activation of NLRP3 by reactive oxygen species (ROS). Activation of the spleen tyrosine kinase SYK was also required for IL- $1 \beta$ secretion and, noting the increased expression of the transmembrane protein SCIMP in monocytes exposed to ApoC3, the researchers suggest that SCIMP might mediate SYK phosphorylation by binding to the tyrosine kinase LYN. "Our work describes a detailed pathway of alternative NLRP3 activation in human monocytes," remarks Speer.

ApoC3 did not induce alternative NLRP3 activation in mouse monocytes, so the researchers used immunodeficient mice reconstituted with human monocytes to examine the effects of ApoC3 in vivo and showed that injection of human ApoC3 induced IL-1 $\beta$ expression. In a model of perivascular carotid injury, injection of ApoC3 compromised vascular regeneration; inhibitors of NLRP3 or of caspase 8 restored re-endothelization of the carotid artery. In the unilateral ureter ligation model of kidney injury, ApoC3 led to a significant increase in the renal monocyte infiltrate and exacerbated renal damage. "These results suggest that triglyceride-rich lipoproteins such as ApoC3, which are present at higher levels in the plasma of patients with CKD than that of healthy individuals, might contribute to organ damage," adds Speer. Among patients undergoing cardiac angiography, high plasma levels of ApoC3 positively correlated with systemic markers of inflammation and, over a follow-up period of 9.9 years, high plasma ApoC3 was also associated with higher all-cause mortality.

"Our findings hint at a novel link between CVD and CKD," notes Speer. "We will now explore whether specific inhibition of these pathways can halt the progression of CKD."

Monica Wang

ORIGINAL ARTICLE Zewinger, S. et al. Apolipoprotein $\mathrm{C} 3$ induces inflammation and organ damage by alternative inflammasome activation. Nat. Immunol. https://doi.org/10.1038/ s41590-019-0548-1 (2019) 